\title{
Prevalencia de sintomáticos respiratorios en instituciones prestadoras de servicios de salud de Bogotá, D.C., junio de 2005 a marzo de 2006
}

\author{
Constanza Collazos ${ }^{1}$, Gabriel Carrasquilla ${ }^{1,2}$, Milcíades Ibáñez ${ }^{3}$, Lilia Edith López ${ }^{4}$ \\ 1 Centro de Estudios e Investigación en Salud, Fundación Santa Fe de Bogotá, Bogotá, D.C., Colombia \\ 2 Universidad del Valle, Facultad de Salud, Cali, Colombia \\ 3 Universidad El Bosque, Facultad de Salud, Bogotá, Colombia \\ ${ }^{4}$ Secretaría Distrital de Salud de Bogotá, Bogotá, Colombia
}

Introducción. Una de las mayores dificultades en el control de la tuberculosis es la detección tardía de casos, lo que obedece, entre otros motivos, a la poca búsqueda activa de casos sintomáticos respiratorios entre los consultantes y al reducido número de baciloscopias ordenados por el personal de salud.

Objetivo. Estimar la prevalencia de casos sintomáticos respiratorios entre usuarios de la red de prestadores de servicios de salud de Bogotá mayores de 15 años, y la proporción a quienes se les solicita baciloscopia de manera oportuna.

Materiales y métodos. Se trató de una encuesta transversal en una muestra probabilística, estratificada, multietápica, de conglomerados sin reemplazo, de 113 instituciones de salud y 3.710 usuarios. Se hizo seguimiento telefónico y revisión de registros de laboratorio para saber a quiénes se les solicitó baciloscopia en la consulta médica.

Resultados. La prevalencia de casos sintomáticos respiratorios en instituciones de salud de Bogotá entre junio de 2005 y marzo de 2006 fue de 7,49\% (IC95\% 6,40-8,59), y fue mayor en instituciones públicas $(9,48 \%)$ (IC95\% 8,04-10,92), en instituciones de primer nivel (8,61\%) (IC95\% 7,40-9,82), en mayores de 60 años $(15,79 \%)$ (IC95\% 12,36-19,23) y en personas afiliadas al Sistema General de Seguridad Social en Salud (7,57\%) (IC95\% 6,42-8,72). No se solicitó baciloscopia al $47 \%$ de los casos sintomáticos respiratorios.

Conclusiones. La proporción de casos sintomáticos respiratorios estimada en este estudio con alta precisión (menos de $2 \%$ de error estándar relativo), es la cifra más actualizada en Colombia y permite hacer inferencias para las instituciones prestadoras de salud de Bogotá, dada la expansión de la muestra.

Se podrán ajustar las metas del programa de control de la tuberculosis en Bogotá, cuya base hasta ahora es un estudio de 1977 (prevalencia de 10\%). Es importante sensibilizar al personal médico para la búsqueda de casos de tuberculosis mediante baciloscopias.

Palabras clave: Mycobacterium tuberculosis, tuberculosis/epidemiología, tuberculosis/diagnóstico, signos y síntomas respiratorios, determinación, servicios de salud.

\section{Prevalence of respiratory symptomatic in health institutions of Bogotá, Colombia}

Introduction. One of the greatest challenges in tuberculosis control is the early detection of cases. Detection is hindered by low level of active search for respiratory symptoms by health consultants and the small number of tubercular smear tests ordered by health personnel.

Objective. The prevalence of individuals with respiratory symptoms was estimated in Bogotá Health Service Institutions, along with the proportion of those receiving diagnostic baciloscopies (smear or culture tests).

Materials and methods. A cross sectional survey was carried out in 113 health service institutions located in the city of Bogotá, involved 3,170 users and covered a 10-month period between June 2005 and March 2006. Sampling design was based on a probabilistic, stratified, multistage, cluster-withoutreplacement strategy. A telephone follow-up and review of laboratory registers was done to identify symptomatic individuals for whom baciloscopy was requested.

Results. The prevalence of symptomatic individuals was $7.5 \%(95 \% \mathrm{Cl} 6.4-8.6 \%)$. A higher prevalence occurred in public institutions, $9.5 \%(95 \% \mathrm{Cl} 8.0-10.9 \%)$, in institutions of first level, $8.6 \%(95 \% \mathrm{Cl} 7.4-$ $9.8)$, in persons $\geq 60$ years of age $15.8 \%(95 \% \mathrm{Cl} 12.4-19.2 \%)$ and in health users with social security, $7.6 \%(95 \% \mathrm{Cl} 6.4-8.7 \%)$. In $47 \%$ of symptomatic cases, a baciloscopic procedures were not ordered.

Conclusions. The study updated the prevalence of respiratory symptomatic individuals in Colombia 
and due to the large sample size (and small confidence interval), inferences can be generalized to the entire health service system in Bogotá. The current tuberculosis program goals in Bogotá require adjustment based on the current prevalence of $7.5 \%$ compared with a 1977 estimate of $10 \%$.. The study recommends an awareness program for physicians to use TB diagnostic tests in suspect cases.

Key words: Mycobacterium tuberculosis, tuberculosis/epidemiology; signs and symptoms, respiratory; tuberculosis/diagnosis, determination, health services

La tuberculosis constituye un problema importante de salud pública en el mundo, a pesar de la disponibilidad de medios para controlarla. El informe mundial de la Organización Mundial de la Salud del 2009 sobre el control de la tuberculosis revela que el porcentaje de la población mundial afectada por la enfermedad alcanzó su valor máximo en 2004, con 142 casos incidentes por 100.000 habitantes; desde entonces, la tasa de incidencia ha disminuido lentamente, a menos de $1 \%$ por año, y presentó un valor de 139 por 100.000 habitantes en el año 2007 (1).

Se estima que en el 2007 hubo 9,27 millones de casos incidentes de tuberculosis a nivel mundial, es decir, más que los $9,24,8,3$ y 6,6 millones registrados en 2006, 2000 y 1990, respectivamente. Los casos prevalentes se estimaron en 13,7 millones y 1,3 millones de muertes y, además, 456.000 muertes en pacientes con virus de la inmuinodeficiencia humana $(\mathrm{VIH})(1)$.

Para el mismo año 2007, en la región de las Américas se registraron 294.636 nuevos casos, para una tasa de incidencia de 32 por 100.000 habitantes; la prevalencia fue de 38 por 100.000 y la mortalidad durante ese año se estimó en 4 por 100.000 (40.616 personas) (1).

En Colombia, en el 2009 se registraron 11.671 casos, de los cuales, 9.632 fueron de tipo pulmonar (con baciloscopia positiva o negativa, recaídas y abandonos, entre otros) y 2.039 fueron extrapulmonares.

La incidencia de tuberculosis en el país es de 25,9 casos por cada 100.000 habitantes. La población en la cual se presentan más casos se encuentra en las edades entre los 25 y 34 años. Sin embargo, la tasa más alta es en personas mayores de 65 años. En este año se presentaron 741 casos en menores

Correspondencia:

Constanza Collazos, Carrera 7A № 123-90, Bogotá, D.C., Colombia.

Teléfono: (+571) 603 0303, extensión 5701; fax: (+571) 214 6668

cocovi@etb.net.co

Recibido: 29/01/10; aceptado:29/06/10 de 15 años; de éstos, 155 eran menores de 5 años. La incidencia específica de tuberculosis en todas las formas en este grupo es de 3,6 por 100.000 habitantes. Los hombres son los más afectados, en todos los grupos de edad (razón de 1,5:1), aunque la diferencia se acentúa a mayor edad. En Colombia existe, aproximadamente, $10 \%$ de casos de infección concomitante tuberculosis y $\mathrm{VIH}$. Las muertes en el 2007 por tuberculosis y sus secuelas fueron de 1.208 casos (2).

Para el 2009, en la red pública y privada de Bogotá se diagnosticaron 1.147 nuevos casos, para una tasa de incidencia de 15,79 por 100.000 habitantes; las formas pulmonares $(60 \%)$ predominan sobre las extrapulmonares (40\%) (2). En estas últimas, la tuberculosis pleural, seguida de la miliar y la ganglionar, es la más frecuente. El sexo más afectado por la tuberculosis es el masculino, con $62 \%$ de los casos. La población afectada se encuentra en su mayor parte en edad económicamente activa, es decir, entre los 15 y los 45 años, pero la incidencia específica por grupo etario es alarmante en los mayores de 65 años. La mayoría de los pacientes, cerca del $70 \%$, se diagnostican en los centros de atención de tercer nivel de la red pública y privada. El comportamiento de la infección concomitante tuberculosis y VIH se mantiene entre los más altos del país $(12,4 \%)$, frente al promedio nacional de 5,8\% (3).

Al comienzo del milenio se suscribieron las metas de la Campaña mundial contra la tuberculosis para el 2005 [cobertura DOTS (Directly Observed Therapy) y diagnóstica de, al menos, $70 \%$ y tasa de curación de, al menos, $85 \%$ ) (4). En 2005, los programas DOTS notificaron cerca de 5 millones de pacientes con tuberculosis en el mundo y se esperaba que el número total de casos diagnosticados y tratados en 2006 se ajustaran al Plan Mundial para Detener la Tuberculosis, 2006-2015, y haber reducido en $50 \%$ la prevalencia y las tasas de mortalidad de la tuberculosis, en comparación con los niveles de 1990 (5). Sin embargo, las cifras mundiales más recientes reportan que se ha logrado solamente el $63 \%$ de la detección de casos y el $85 \%$ de curación (1). El cumplimiento de estas metas es un paso 
muy importante para alcanzar las metas del milenio en el 2015.

Una de las mayores dificultades en el control de la tuberculosis es la detección temprana de casos, para lo cual es necesario identificar los casos sintomáticos respiratorios. En Bogotá, el diagnóstico se hace principalmente en las instituciones de tercer nivel de atención (70\% de los casos) y en estados avanzados de la enfermedad; este diagnóstico tardío obedece a la poca búsqueda activa de pacientes sintomáticos respiratorios entre los consultantes y al reducido número de baciloscopias ordenados por el personal de salud, según el documento "Implementación de la estrategia tratamiento acortado estrictamente supervisado en el Distrito Capital, 2004", elaborado por el referente del programa de enfermedades trasmisibles de la Dirección de Salud Pública de Bogotá.

El Programa Nacional de Tuberculosis y los programas de las secretarías de salud en el país programaban sus metas y actividades con base en un estudio realizado en 1977 en el departamento de Risaralda en 5.530 pacientes mayores de 10 años que asistían por primera vez a la consulta de los servicios de salud. Esta investigación reportó una prevalencia de casos sintomáticos respiratorios de $12,6 \%$ en el departamento y de $10,3 \%$ en la Pereira (6). No existen otros estudios en el país sobre prevalencia de sintomáticos respiratorios en instituciones de salud, con representatividad para población que consulta a estos servicios.

Un estudio de la Fundación Santa Fe de Bogotá y la Secretaría de Salud en 1999, reportó una prevalencia de sintomáticos respiratorios en la población general de 0,9\% (7). En el 2001, se hizo un estudio en el municipio de Mitú, Vaupés, un área muy endémica para tuberculosis en Colombia, en el que se encontró una prevalencia de sintomáticos respiratorios en la población general de 3,6\% (IC95\% 2,4-4,9\%) (8). En 2006 se llevó a cabo un estudio en cuatro hospitales de tercer nivel de Bogotá seleccionados por conveniencia, en el que se encontró una prevalencia de sintomáticos respiratorios de 48,5\% (9).

No encontramos en la literatura científica reportes de estudios de prevalencia de casos sintomáticos respiratorios en instituciones de salud en otros países. Es necesario contar con cifras actualizadas para efectos de la planeación de actividades de detección, diagnóstico y tratamiento del programa de tuberculosis; por esta razón, se realizó un estudio para estimar la prevalencia de sintomáticos respiratorios entre los usuarios mayores de 15 años de la red de prestadores de salud del Distrito Capital.

\section{Materiales y métodos}

\section{Tipo de estudio}

Se hizo un estudio de corte transversal en los usuarios mayores de 15 años que consultaron por primera vez en el año a la consulta externa en alguna de las instituciones prestadoras de servicios de salud (IPS) de Bogotá, entre junio de 2005 y marzo de 2006.

\section{Diseño y tamaño de la muestra}

El marco muestral lo constituyeron 540 IPS públicas y privadas registradas en la base de datos de la Secretaría de Salud al 31 de mayo de 2005.

Se diseñó una muestra probabilística, estratificada, multietápica, de conglomerados sin reemplazo, en la que se conformaron estratos por localidad (19 localidades), tipo de institución de salud (pública o privada) y nivel de complejidad (I, II y III).

Se consideraron las instituciones como unidades primarias de muestreo, buscando la máxima homogeneidad dentro de cada estrato y la máxima heterogeneidad entre los mismos.

Las instituciones se seleccionaron en forma aleatoria, con ponderación por número de usuarios (asignación proporcional). Los conglomerados que se utilizaron fueron el hospital (unidad primaria de muestreo), que estaba conformado por el conjunto de consultorios y a su vez de pacientes, y el consultorio (unidad secundaria de muestreo), conformado por el conjunto de pacientes (unidad terciaria de muestreo). En aquellas IPS en las que había más de un consultorio, se seleccionó el consultorio o los consultorios de manera aleatoria y luego se pasó a la identificación de los usuarios para la encuesta, seleccionados al azar de la lista de consulta del día.

Se obtuvo un tamaño de muestra de 3.038 pacientes, teniendo en cuenta una prevalencia de sintomáticos respiratorios de 1\% (7), una precisión de $0,5 \%$ (e=|P-p|=0,005), un error estándar relativo $\left(e_{r}\right)$ esperado entre $15,1 \%$ y $30 \%$ (considerados como aceptables según los parámetros internacionales de investigación estadística de Canadá) (10), una confiabilidad del $95 \%$ y un efecto de diseño por conglomerados (deff) de 2. Ajustando por un porcentaje de falta de respuesta del $20 \%$, el tamaño final de muestra fue de 3.798 pacientes. 


\section{Definición de sintomático respiratorio}

De acuerdo con la definición de la Organización Mundial de la Salud (OMS) (11), se consideró sintomático respiratorio a toda persona con tos $y$ expectoración de más de 15 días de evolución.

\section{Criterios de inclusión}

Se seleccionaron usuarios que tuvieran más de 15 años, que hubieran consultado por primera vez en el año, que nunca hubieran tenido diagnóstico de tuberculosis, que hubieran residido en la ciudad durante el último mes y que aceptaran contestar la encuesta.

\section{Recolección de información}

Para obtener datos sobre síntomas respiratorios y características de los usuarios, se diseñó una encuesta estructurada conservando las variables de la encuesta utilizada en el estudio de 1999, con el fin de poder hacer comparaciones posteriores. En la primera hoja de la encuesta se incluyó el consentimiento informado para ser diligenciado antes de obtener la información.

Se incluyeron: variables sociodemográficas (edad, sexo, raza, estado civil, personas con las que vive, ocupación educación, estrato socioeconómico, nivel de sistema de identificación de potenciales beneficiarios de programas sociales -SISBEN-, afiliación al Sistema General de Seguridad Social en Salud, tipo de afiliación y tipo de afiliado); datos clínicos generales (motivo de consulta, por iniciativa de quién consulta, antecedentes de tuberculosis como criterio de exclusión del estudio, presencia de síntomas generales y vacunación con BCG); historia y características de la tos y el esputo (cuándo le da, desde cuándo la tiene, si se acompaña de flemas, si hay hemoptisis); hábitos (tabaquismo, cocina con leña o carbón, consumo de sustancias psicotrópicas, prácticas homosexuales, ejercicio físico); enfermedades asociadas (enfermedad diagnosticada, cirugías previas, medicamentos inmunosupresores); y de seguimiento de los casos sintomáticos respiratorios para verificar la sospecha médica de sintomatología respiratoria y solicitud de baciloscopias.

Se realizó una prueba piloto del instrumento en tres instituciones (una de cada nivel) en las que se aplicaron 28 encuestas. Basados en estos resultados, se ajustó el instrumento, y se elaboraron los manuales de capacitación y el folleto instructivo para la el operativo de campo.
Para la recolección de la información, se capacitó un grupo de 10 encuestadoras durante dos días, en contenidos básicos de tuberculosis y de la encuesta, la forma de hacer las preguntas, el comportamiento en las IPS y las normas éticas de la encuesta a los usuarios.

\section{Manejo y análisis de los datos}

La digitación de los datos se realizó en el programa Ep-Info 2000 (12). Se aplicaron programas de verificación automática de digitación para depurar la información (CHECK) y se procesó la información en el paquete estadístico STATA®, versión 8 (13).

Se evaluó la precisión de la estimación de la prevalencia de casos sintomáticos respiratorios mediante el error estándar y el error estándar relativo, esperando que fuera menor de 10\%. Este cálculo se hizo en CLUSTER y SUDAN, teniendo en cuenta el diseño probabilístico, multietápico, estratificado, por conglomerados, y los pesos de ponderación o de inferencia a la población diana o blanco (target) originados del marco muestral de los hospitales de la red prestadora de servicios de Bogotá (pública y privada).

También, se midió la eficiencia de las estimaciones de la muestra obtenida mediante el efecto del diseño, y la estimación de casos sintomáticos respiratorios se realizó mediante la probabilidad del modelo de frecuencia con su respectivo intervalo de confianza del $95 \%$.

La precisión de las estimaciones se evaluó a partir del error estándar relativo, teniendo en cuenta los estándares de calidad del Canadá, que establecen la calidad A, como buena precisión de las estimaciones, entre $0 \%$ y $15 \%$; la calidad B, como precisión aceptable, entre $15,1 \%$ y $30 \%$; la calidad C, entre 30,1 y $50 \%$ (son estimaciones que deben interpretarse con precaución); y la calidad D, más de $50 \%$ de los que no es posible hacer alguna inferencia.

Se hicieron análisis descriptivos de las frecuencias de casos sintomáticos respiratorios, en los cuales se utilizaron distribuciones de frecuencias y porcentajes de tendencia central y medidas de dispersión.

\section{Control de calidad de la información}

Las encuestas recolectadas se recibían tres veces por semana en la oficina del Centro de Estudios e Investigación en Salud; se hacía una crítica cruzada de las encuestas, se identificaban las fallas, se 
reforzaba la capacitación y, de ser necesario, se repetían las encuestas. Además, semanalmente se hacía verificación telefónica de la información al $10 \%$ de las encuestas y se hizo doble digitación para garantizar la calidad final. El protocolo del estudio fue aprobado por el Comité de Ética de la Fundación Santa Fe de Bogotá.

\section{Resultados}

Se recolectó información de 113 IPS, 19 de las 20 localidades de Bogotá; se excluyó la localidad de Sumapaz, porque no ha reportado casos de tuberculosis desde el año 2000. Se aplicaron 4.000 encuestas a usuarios de IPS públicas y privadas de niveles I, II y III. La tasa de falta de respuesta fue de 5,7\%, particularmente, en instituciones privadas y en personas de mayores estratos sociales. Se descartaron 72 encuestas porque no cumplían con los criterios de inclusión del estudio. La muestra final fue de 3.710 personas encuestadas.

De las IPS de la muestra, 34,5\% eran entidades públicas y $65,5 \%$ privadas. Según el nivel de complejidad de atención, el $67,3 \%$ de las encuestas se obtuvieron en instituciones de primer nivel, el $13,2 \%$ en segundo nivel y el $19,5 \%$ en tercer nivel.

\section{Características sociodemográficas de los usuarios de IPS}

Las características sociodemográficas y las condiciones de uso de servicios de los usuarios entrevistados, se presentan en el cuadro 1.

Estado clínico general

Motivo de consulta. La mayoría de los usuarios acuden al servicio de salud porque se sienten enfermos; el $26,5 \%$ acude para un chequeo médico anual.

Por iniciativa de quién consulta. El $90 \%$ de los usuarios acude por iniciativa propia y sólo el $8 \%$ es remitido por un médico o por otra IPS.

En el cuadro 2 se presentan la frecuencia y el porcentaje de usuarios que refirieron síntomas generales en las dos últimas semanas.

Vacunación con BCG. Refirieron haber recibido vacunación con BCG 2.532 usuarios (68,25\%), $593(15,98 \%)$ dijeron no haberla recibido y 585 $(15,77 \%)$ no recordaban haber sido vacunados. En cuanto a la cicatriz de BCG, 2.415 (65,09\%) usuarios la presentaban, $896(24,15 \%)$ no la presentaban y $399(10,75 \%)$ no mostraron el brazo. Se encontró un alta concordancia entre los
Cuadro 1. Características sociodemográficas de los usuarios de IPS, Bogotá, D.C., junio de 2005 a marzo de 2006.

\begin{tabular}{|c|c|c|}
\hline $\begin{array}{l}\text { Características } \\
\text { sociodemográficas }\end{array}$ & $\begin{array}{l}\text { Frecuencia } \\
(n=3.710)\end{array}$ & Porcentaje \\
\hline \multicolumn{3}{|l|}{ 1. Sexo } \\
\hline Masculino & 1.083 & 29,19 \\
\hline Femenino & 2.627 & 70,81 \\
\hline \multicolumn{3}{|l|}{ 2. Edad (años) } \\
\hline $15-19$ & 285 & 7,68 \\
\hline $20-29$ & 921 & 24,82 \\
\hline $30-39$ & 835 & 22,51 \\
\hline $40-49$ & 635 & 17,12 \\
\hline $50-59$ & 462 & 12,45 \\
\hline $60 y+$ & 572 & 15,42 \\
\hline \multicolumn{3}{|l|}{ 3. Raza } \\
\hline Blanca & 701 & 18,89 \\
\hline Mestiza & 2.930 & 78,98 \\
\hline Negra & 73 & 1,97 \\
\hline Indígena & 6 & 0,16 \\
\hline Rom (gitanos) & 0 & 0 \\
\hline \multicolumn{3}{|l|}{ 4. Estado civil } \\
\hline Soltero & 1.116 & 30,08 \\
\hline Casado & 1.223 & 32,96 \\
\hline Unión libre & 907 & 24,45 \\
\hline Separado - Divorciado & 244 & 6,58 \\
\hline Viudo & 220 & 5,93 \\
\hline \multicolumn{3}{|l|}{ 5. Educación } \\
\hline Ninguna & 98 & 2,64 \\
\hline Primaria incompleta & 428 & 11,53 \\
\hline Primaria completa & 568 & 15,31 \\
\hline Secundaria incompleta & 731 & 19,70 \\
\hline Secundaria completa & 1.026 & 27,65 \\
\hline Técnica/Universitaria & 825 & 22,23 \\
\hline Sin información & 34 & 0,92 \\
\hline \multicolumn{3}{|l|}{ 6. Ocupación } \\
\hline Empleado & 1.488 & 40,11 \\
\hline Independiente & 302 & 8,14 \\
\hline Retirado/pensionado & 109 & 2,94 \\
\hline Ama de casa/hogar & 1.400 & 37,74 \\
\hline Desempleado & 92 & 2,48 \\
\hline Estudiante & 314 & 8,46 \\
\hline Religioso & 5 & 0,13 \\
\hline \multicolumn{3}{|l|}{ 7. Estrato socioeconómico } \\
\hline 1,2 y 3 & 3320 & 89,49 \\
\hline 4,5 y 6 & 390 & 10,51 \\
\hline \multicolumn{3}{|c|}{ 8. Número de residentes por hogar } \\
\hline 1 & 94 & 2,54 \\
\hline $2-4$ & 2.675 & 72,10 \\
\hline $5 y+$ & 941 & 25,36 \\
\hline \multicolumn{3}{|c|}{ 9. Le han hecho encuesta SISBEN } \\
\hline Sí & 1.513 & 40,78 \\
\hline No & 2.197 & 59,22 \\
\hline \multicolumn{3}{|l|}{ 10. Nivel de SISBEN } \\
\hline 1 у 2 & 1.283 & 84,80 \\
\hline 3 у 4 & 230 & 15,20 \\
\hline No aplica & 2.197 & 59,22 \\
\hline \multicolumn{3}{|c|}{$\begin{array}{l}\text { 11. Afiliación al Sistema General de } \\
\text { Seguridad Social en Salud }\end{array}$} \\
\hline No & 243 & 6,55 \\
\hline Sí & 3.467 & 93,45 \\
\hline \multicolumn{3}{|c|}{ 12. Tipo de régimen de afiliación } \\
\hline Contributivo & 2.302 & 62,05 \\
\hline Subsidiado & 1.165 & 31,40 \\
\hline Ninguno & 243 & 6,55 \\
\hline
\end{tabular}


Cuadro 2. Frecuencia de usuarios que refirieron síntomas generales en las dos semanas previas a la encuesta, Bogotá, D.C, junio de 2005 a marzo de 2006.

\begin{tabular}{|c|c|c|c|c|c|}
\hline \multirow[t]{2}{*}{ Síntoma } & \multicolumn{2}{|c|}{ No } & \multicolumn{2}{|c|}{ Sí } & \multirow[t]{2}{*}{ Total } \\
\hline & $\mathbf{n}$ & $\%$ & $\mathbf{n}$ & $\%$ & \\
\hline Fiebre & 3.352 & 90,35 & 358 & 9,65 & 3.710 \\
\hline Sudoración & 3.365 & 90,70 & 345 & 9,30 & 3.710 \\
\hline Pérdida de peso & 3.371 & 90,86 & 339 & 9,14 & 3.710 \\
\hline Pérdida de apetito & 3.303 & 89,03 & 407 & 10,97 & 3.710 \\
\hline
\end{tabular}

usuarios que dijeron haber sido vacunados con BGC y aquellos que efectivamente presentaban cicatriz por vacunación (concordancia observada de $0,83, \kappa=0,66, S=0,012, z=54$ y $p=0,000$ ).

Historia y características de la tos y el esputo. El 25,26\% (937) de los usuarios entrevistados refirieron tos en las últimas dos semanas; de los usuarios que refirieron tos, $42 \%$ dijeron haberla tenido todos los días, $40 \%$ algunos días en la semana y $18 \%$ rara vez. De los usuarios con tos, $49,5 \%$ refirió tenerla hacía menos de dos semanas, $27,2 \%$ más de dos semanas pero menos de un mes, $11,5 \%$ de un mes a menos de seis meses, $3,7 \%$ de seis meses a un año y $8,1 \%$ por más de un año.

Hábitos. De las personas que tosen actualmente, $19,74 \%$ son fumadoras. El cuadro 3 muestra la frecuencia de algunos hábitos de los usuarios.

Enfermedades asociadas. La mitad de los usuarios no presentan enfermedades asociadas. En los que presentan alguna enfermedad, la hipertensión era la más frecuente. En el cuadro 4 se presenta la morbilidad de los usuarios entrevistados, según el diagnóstico médico al momento de la encuesta.

Consumo de de medicamentos. Sesenta y tres usuarios $(1,69 \%)$ recibían esteroides, tres usuarios, fármacos para el sida, y 19, para el tratamiento del cáncer. El 15,82\% recibía un medicamento diferente a los anteriores.

\section{Prevalencia de casos sintomáticos respiratorios}

La prevalencia cruda de sintomáticos respiratorios en las IPS de Bogotá fue de 7,49\%, (IC95\% 6,408,59 ; con un error estándar relativo de 7,46 ) y la prevalencia de sintomáticos respiratorios fue mayor en instituciones públicas (cuadro 5).

En la evaluación de la precisión de las estimaciones puntuales y de intervalo (intervalo de confianza del $95 \%$ ) de la prevalencia, se observó una altísima precisión, menor de $2 \%$ (calidad A), y, por lo tanto,
Cuadro 3. Frecuencia de hábitos de los usuarios de IPS, Bogotá, D.C., junio de 2005 a marzo de 2006.

\begin{tabular}{lcc}
\hline Hábito & $\begin{array}{c}\text { Frecuencia } \\
(\mathbf{n = 3 . 7 1 0 )}\end{array}$ & Porcentaje \\
\hline 1. Tabaquismo actual & 590 & 15,90 \\
2. Tabaquismo anterior & 890 & 23,99 \\
3. Cocina con leña o carbón & 117 & 3,15 \\
4. Consumo de licor & 1.505 & 40,57 \\
5. Se emborracha & 403 & 10,86 \\
6. Consumo de sustancias & & 3,28 \\
psicoactivas & 122 & 57,11 \\
\hline
\end{tabular}

Cuadro 4. Morbilidad de los usuarios de IPS, Bogotá, D.C., junio de 2005 a marzo de 2006.

\begin{tabular}{lcc}
\hline Enfermedad & $\begin{array}{c}\text { Frecuencia } \\
(\mathbf{n = 3 . 7 1 0 )}\end{array}$ & Porcentaje \\
\hline 1. Neumonía/pulmonía & 39 & 1,05 \\
2. EPOC/enfisema & 27 & 0,73 \\
3. Bronquitis crónica & 53 & 1,43 \\
4. Asma & 91 & 2,45 \\
5. Fibrosis pulmonar & 10 & 0,27 \\
6. Falla renal/insuficiencia renal & 16 & 0,43 \\
7. Diabetes & 114 & 3,08 \\
8. Artritis reumatoidea & 78 & 2,10 \\
9. Lupus eritematoso & 7 & 0,19 \\
10. Leucemia & 7 & 0,19 \\
11. Linfoma & 3 & 0,08 \\
12. Otro tipo de cáncer & 18 & 0,49 \\
13. VIH/sida & 4 & 0,11 \\
14. Hipertensión & 441 & 11,89 \\
15. Insuficiencia cardiaca & 44 & 1,19 \\
16. Infarto del miocardio & 26 & 0,70 \\
17. Cardiopatía & 59 & 1,59 \\
18. Otra enfermedad & 730 & 19,68 \\
19. Ninguna & 1.929 & 51,29 \\
\hline
\end{tabular}

se puede inferir a las IPS de Bogotá. También, se generaron errores estándar relativos con alta precisión por tipo de IPS (menor de 3\%) y, por lo tanto, la estimación de sintomático respiratorio es se puede inferir para IPS públicas y privadas. Se obtuvieron estimaciones con calidad A, por nivel de complejidad, sexo, edad, estado civil, ocupación, estrato socioeconómico, SISBEN, nivel de SISBEN y afiliación del paciente (cuadro 6). 
Cuadro 5. Frecuencia y prevalencia de sintomáticos respiratorios según tipo de IPS, Bogotá, D.C., junio de 2005 a marzo de 2006

\begin{tabular}{lcccc}
\hline & \multicolumn{4}{c}{ Sintomáticos respiratorios } \\
\cline { 2 - 5 } $\begin{array}{l}\text { Tipo de } \\
\text { IPS }\end{array}$ & $\begin{array}{c}\text { Total } \\
\text { encuestas }\end{array}$ & $\begin{array}{c}\text { Frecuencia } \\
\text { (n) }\end{array}$ & Prevalencia & IC 95\% \\
\hline Pública & 1.091 & 103 & 9.48 & $8,04-10,92$ \\
Privada & 2.619 & 163 & 6.68 & $5,29-8,07$ \\
Total & 3.710 & 266 & 7.49 & $6,40-8,59$ \\
\hline
\end{tabular}

La prevalencia fue mayor en las IPS de primer nivel, en las personas mayores de 60 años, en las que vivían solas y en las afiliadas al Sistema general de seguridad social en salud (figura 1).

\section{Proporción de sintomáticos respiratorios a quienes se les practica baciloscopia diagnóstica}

Por las dificultades administrativas y éticas que representa la revisión de historias clínicas en las IPS, particularmente en las privadas, y para cumplir con este objetivo, se rastreó en el laboratorio de cada IPS o en el laboratorio al cual refieren las muestras y los pacientes, si se habían realizado baciloscopias a los 266 pacientes identificados como sintomáticos respiratorios, por medio de la encuesta del estudio.

Cuando no se encontraron registros de baciloscopias en el laboratorio respectivo, se llamó telefónicamente a estos usuarios y se les preguntó si les habían solicitado dicho examen. Sólo en $8 \%$ de los casos de sintomáticos respiratorios se solicitó un examen diagnóstico $(7,51 \%$ baciloscopia y $0,37 \%$ radiografía). No se solicitó baciloscopia en $47 \%$ (125 personas) y en $45 \%$ no se encontraron registros de laboratorio, siendo esto un importante problema para el sistema de salud.

\section{Discusión}

Los programas de control de la tuberculosis se basan principalmente en la identificación de casos nuevos mediante la búsqueda activa de pacientes sintomáticos respiratorios en comunidades cerradas, la búsqueda pasiva en aquellos pacientes sintomáticos que asisten a consulta o mediante el estudio de los contactos de pacientes con tuberculosis. En Colombia, el Ministerio de la Protección Social utiliza, como meta operativa del programa de prevención y control de la tuberculosis, la identificación de $10 \%$ de casos sintomáticos respiratorios entre los consultantes por primera vez en el año, con base en la cifra reportada en 1977 por Arciniegas et al. (6). La prevalencia encontrada en este estudio $(7,49 \%)$ es menor que la reportada en las instituciones de salud hace 30 años.

De acuerdo con los estándares establecidos, los errores de muestreo están en el nivel de calidad A, lo cual indica que la inferencia en la población estudiada es válida y que los resultados se pueden aplicar a la población que consulta a las IPS de Bogotá, excepto en algunas pocas variables, como otras razas diferentes a la blanca, mestiza o negra, estrato socioeconómico VI y nivel 4 de SISBEN.

Nuestros resultados constituyen una actualización de la cifra de prevalencia de casos sintomáticos respiratorios en las IPS en Bogotá y servirá para ajustar las metas del programa; además, podrá tomarse como referencia para otras ciudades del país y de América Latina.

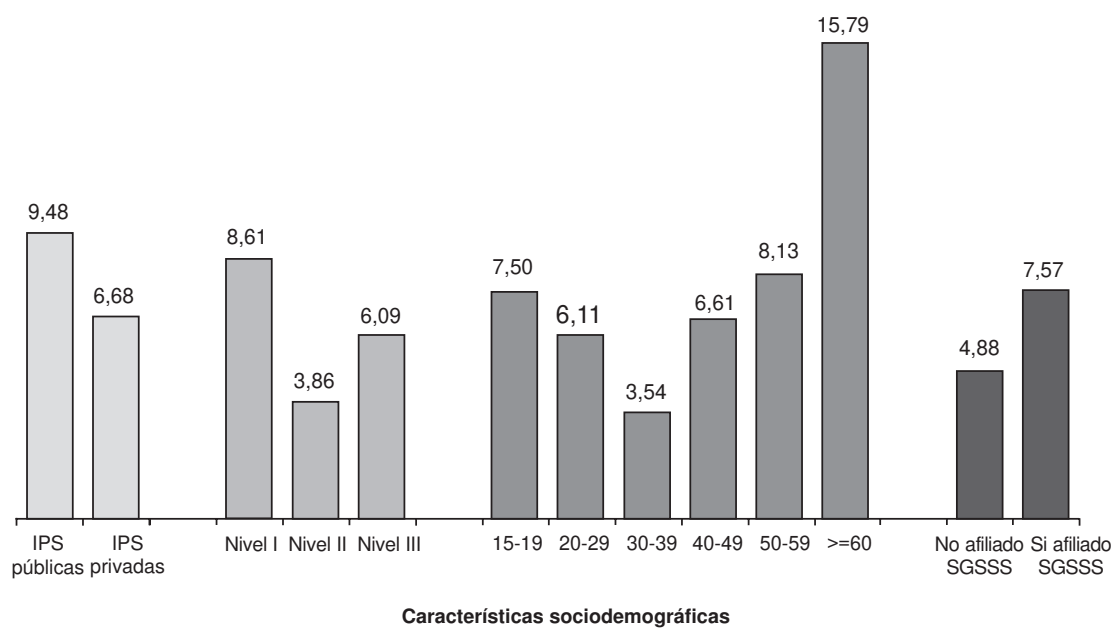

Figura 1. Prevalencia de sintomáticos respiratorios según características sociodemográficas. Bogotá D.C., junio 2005 a marzo 2006. 
Cuadro 6. Estimación de prevalencia de paciente sintomático respiratorio y errores de muestreo, según características sociodemográficas, en IPS de Bogotá, D.C., junio de 2005 a marzo de 2006.

\begin{tabular}{|c|c|c|c|c|c|c|}
\hline \multirow[b]{2}{*}{ Variable } & \multirow[b]{2}{*}{$\begin{array}{l}\text { Prevalencia } \\
\text { (\%) }\end{array}$} & \multirow[b]{2}{*}{$\begin{array}{c}\text { Error } \\
\text { estándar }\end{array}$} & \multirow[b]{2}{*}{$\begin{array}{c}\text { Error } \\
\text { estándar } \\
\text { relativo }\end{array}$} & \multicolumn{2}{|c|}{ IC 95\% } & \multirow[b]{2}{*}{$\begin{array}{l}\text { Efecto de } \\
\text { diseño }\end{array}$} \\
\hline & & & & $\begin{array}{l}\text { Límite } \\
\text { inferior }\end{array}$ & $\begin{array}{c}\text { Límite } \\
\text { superior }\end{array}$ & \\
\hline $\begin{array}{l}\text { Sintomático respiratorio } \\
\text { 1. Tipo de IPS }\end{array}$ & 7,499 & 0,560 & 7,468 & 6,400 & 8,597 & 1,679 \\
\hline Pública & 9,486 & 0,734 & 7,738 & 8,048 & 10,925 & 0,684 \\
\hline Privada & 6,683 & 0,710 & 10,624 & 5,290 & 8,075 & 2,120 \\
\hline \multicolumn{7}{|l|}{ 2. Nivel de complejidad } \\
\hline I & 8,613 & 0,619 & 7,187 & 7,401 & 9,825 & 1,071 \\
\hline II & 3,863 & 1,195 & 30,935 & 1,521 & 6,206 & 0,746 \\
\hline III & 6,090 & 0,907 & 14,893 & 4,311 & 7,868 & 1,891 \\
\hline \multicolumn{7}{|l|}{ 3. Sexo } \\
\hline Mujeres & 7,255 & 0,733 & 10,103 & 5,818 & 8,691 & 2,097 \\
\hline Hombres & 8,089 & 0,688 & 8,505 & 6,741 & 9,437 & 0,689 \\
\hline \multicolumn{7}{|l|}{ 4. Edad (años) } \\
\hline $15-19$ & 7,500 & 2,090 & 27,867 & 3,403 & 11,597 & 1,795 \\
\hline $20-29$ & 6,112 & 1,024 & 16,754 & 4,105 & 8,119 & 1,682 \\
\hline $30-39$ & 3,542 & 0,540 & 15,246 & 2,483 & 4,600 & 0,713 \\
\hline $40-49$ & 6,616 & 0,684 & 10,339 & 5,274 & 7,957 & 0,481 \\
\hline $50-59$ & 8,136 & 1,075 & 13,213 & 6,030 & 10,242 & 0,714 \\
\hline$\geq 60$ & 15,798 & 1,752 & 11,090 & 12,363 & 19,232 & 1,320 \\
\hline \multicolumn{7}{|l|}{ 5. Raza } \\
\hline Blanca & 7,297 & 1,417 & 19,419 & 4,519 & 10,075 & 2,081 \\
\hline Mestiza & 7,527 & 0,510 & 6,776 & 6,527 & 8,526 & 1,094 \\
\hline Negra & 7,521 & 2,596 & 34,517 & 2,432 & 12,609 & 0,707 \\
\hline Otra raza & $\star * *$ & & & & & \\
\hline \multicolumn{7}{|l|}{ 6. Estado civil } \\
\hline Soltero & 7,411 & 0,935 & 12,616 & 5,578 & 9,243 & 1,421 \\
\hline Casado & 7,779 & 0,857 & 11,017 & 6,098 & 9,459 & 1,253 \\
\hline Unión libre & 5,789 & 0,771 & 13,318 & 4,278 & 7,300 & 0,988 \\
\hline Separado/ Divorciado & 8,826 & 1,705 & 19,318 & 5,484 & 12,168 & 0,882 \\
\hline Viudo & 11,646 & 2,504 & 21,501 & 6,739 & 16,553 & 1,340 \\
\hline \multicolumn{7}{|l|}{ 7. Educación } \\
\hline Ninguna & 14,319 & 4,534 & 5,119 & 5,434 & 23,205 & 1,642 \\
\hline Primaria incompleta & 14,351 & 1,667 & 21,819 & 11,084 & 17,618 & 0,967 \\
\hline Primaria completa & 8,686 & 0,833 & 17,800 & 7,053 & 10,320 & 0,497 \\
\hline Secundaria incompleta & 6,813 & 1,237 & 17,687 & 4,388 & 9,239 & 1,763 \\
\hline Secundaria completa & 5,982 & 0,763 & 22,079 & 4,486 & 7,477 & 1,062 \\
\hline Técnico o universitaria & 4,628 & 0,743 & 13,872 & 3,172 & 6,084 & 1,032 \\
\hline \multicolumn{7}{|l|}{ 8. Ocupación } \\
\hline Empleado & 5,483 & 0,613 & 11,180 & 4,282 & 6,685 & 1,079 \\
\hline Independiente & 12,307 & 1,554 & 12,627 & 9,262 & 15,352 & 0,675 \\
\hline Retirado/pensionado & 8,584 & 1,967 & 22,915 & 4,728 & 12,441 & 0,538 \\
\hline Ama de casa/hogar & 8,125 & 0,881 & 10,843 & 6,399 & 9,852 & 1,452 \\
\hline Desempleado & 10,144 & 2,774 & 27,346 & 4,708 & 15,581 & 0,776 \\
\hline Estudiante & 8,760 & 3,306 & 37,740 & 2,280 & 15,240 & 4,294 \\
\hline Religioso & $* * *$ & & & & & \\
\hline \multicolumn{7}{|l|}{ 9. Estrato socioeconómico } \\
\hline 1 & 8,735 & 1,444 & 16,531 & 5,904 & 11,565 & 1,015 \\
\hline 2 & 8,053 & 0,684 & 8,494 & 6,712 & 9,394 & 1,023 \\
\hline 3 & 6,552 & 0,772 & 11,783 & 5,041 & 8,067 & 1,278 \\
\hline 4 & 8,904 & 2,249 & 25,258 & 4,496 & 13,311 & 1,833 \\
\hline 5 & $* * *$ & & & & & \\
\hline 6 & $* * *$ & & & & & \\
\hline \multicolumn{7}{|c|}{ 10. Número de residentes por hogar } \\
\hline 1 & 17,447 & 5,252 & 6,125 & 7,154 & 27,741 & 1,800 \\
\hline $2-4$ & 5,597 & 0,933 & 8,330 & 3,769 & 7,425 & 0,710 \\
\hline $5-10$ & 6,916 & 0,870 & 16,509 & 5,211 & 8,622 & 0,782 \\
\hline $11 y+$ & 7,292 & 1,354 & 23,279 & 4,638 & 9,946 & 2,371 \\
\hline \multicolumn{7}{|l|}{ 11. SISBEN } \\
\hline No & 7,042 & 0,686 & 9,742 & 5,697 & 8,388 & 1,549 \\
\hline
\end{tabular}




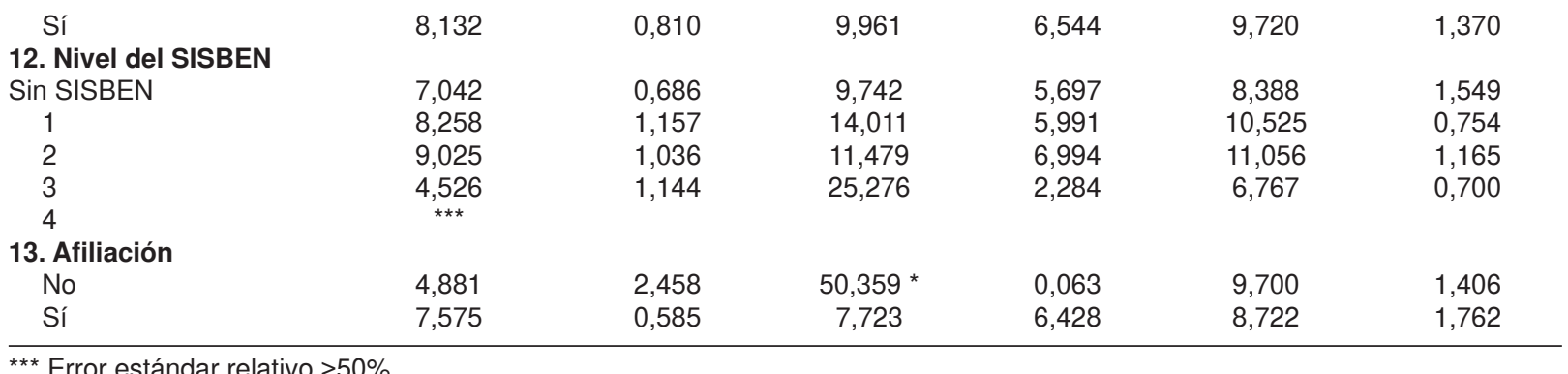

Los resultados más recientes en el país han reportado prevalencia de casos de sintomáticos respiratorios de $0,9 \%$ en la población general de Bogotá (7) y de 3,6\% en la población general de Mitú (8), pero no son comparables con la cifra encontrada en este estudio, realizado en instituciones de salud. La prevalencia de $48,5 \%$, encontrada por Henao et al. (9) en cuatro hospitales de tercer nivel en la ciudad de Bogotá, se basó en una muestra por conveniencia de hospitales; por lo tanto, tampoco es comparable con los hallazgos aquí reportados, que tienen representatividad para todas las IPS de Bogotá en los tres niveles de atención y en todas las localidades de la ciudad.

Entre los países latinoamericanos, Perú reporta una prevalencia de $5 \%$ de pacientes sintomáticos respiratorios en la población general a nivel nacional (14).

Se encontró una prevalencia significativamente mayor en las IPS públicas que en las privadas, hallazgo acorde con lo esperado, considerando que a estas instituciones acuden más personas de escasos recursos; la pobreza se reconoce como un factor de riesgo para la tuberculosis (15). Por otra parte, la prevalencia fue mayor en las instituciones de primer nivel, lo que se explica porque éstas constituyen la puerta de entrada del sistema de salud.

En este estudio no encontramos diferencias significativas de prevalencia de pacientes sintomáticos respiratorios entre hombres y mujeres, aunque se reconoce que la tuberculosis es una enfermedad que afecta principalmente a los hombres $(16,17)$. En la literatura disponible no se reportan diferencias de sexo y sintomatología respiratoria, pero se mencionan 1,8 casos de tuberculosis en hombres anualmente, en relación con un caso en mujeres (18). Algunos hallazgos sugieren que esto puede deberse a desigualdades en el acceso a la atención, en detrimento de la salud de las mujeres, o puede reflejar diferencias epidemiológicas en tuberculosis entre hombres y mujeres (18).
Respecto al grupo de edad, las personas mayores de 60 años presentaron la mayor prevalencia como sintomáticos respiratorios, aunque los más recientes reportes en la literatura mencionan que la tasa de incidencia de tuberculosis es mayor en adultos jóvenes (15). Las personas mayores pueden presentar sintomatología respiratoria asociada a otras entidades, como tabaquismo, enfermedad pulmonar o enfermedades cardiovasculares.

En este estudio puede existir una subestimación de la prevalencia de pacientes sintomáticos respiratorios por varias razones. Encontramos que 93\% de los sujetos incluidos en el estudio reportó estar afiliado al Sistema General de Seguridad Social en Salud, lo cual puede indicar que los no afiliados (en su mayoría población pobre) tienen limitaciones para el acceso a los servicios y, como se anotó antes, están en mayor riesgo de tener tuberculosis (19). En Colombia se observó un estancamiento de las tasas de egresos hospitalarios y de mortalidad por tuberculosis a partir de 1990, que pueden explicarse por los cambios del sistema de salud y por un manejo tardío por parte de los servicios de salud; el acceso diferencial a los servicios puede estar originando una disminución de los casos diagnosticados (20-22).

No se encontraron pacientes sintomáticos respiratorios con $\mathrm{VIH}$, aunque diversos estudios tanto nacionales como internacionales demuestran que existe una asociación entre tuberculosis y VIH (23-25). Los bajos registros de casos de infección concomitante se atribuyen, entre otras, a las fallas de los sistemas de información en el país. Durante el 2005, sólo 11 de las 36 entidades territoriales reportaron casos de infección concomitante (26). Además, algunos pacientes no mencionan la infección de $\mathrm{VIH}$ por el estigma relacionado con esta condición y, por lo tanto, pudo presentarse subestimación en la presencia simultánea de sintomatología respiratoria y $\mathrm{VIH}$.

La dificultad administrativa y ética que presenta la revisión de historias clínicas en las IPS constituyó 
el principal obstáculo para estimar el porcentaje de pacientes a quienes el médico les hace diagnóstico de sintomático respiratorio y les solicita baciloscopia, particularmente en las instituciones privadas. La falta de sospecha del médico sobre esta sintomatología, y sobre la enfermedad, constituye una importante limitación en la identificación de casos de sintomáticos respiratorios $\mathrm{y}$, por lo tanto, de pacientes con tuberculosis pulmonar (27). El porcentaje de captación de sintomáticos respiratorios en las consultas médicas por todas las causas, en mayores de 15 años en el país, se redujo en $48,7 \%$ entre 2001 y 2002 (28).

Este estudio constituye una importante fuente de información para la actualización de las cifras de pacientes sintomáticos respiratorios esperados en las instituciones de salud en Bogotá; la rigurosidad del proceso de muestreo y de la selección de IPS y usuarios, permite contar con cifras confiables que brindan parámetros, tanto a nivel nacional como internacional, para el análisis de las políticas relacionadas con el control de la tuberculosis y para la programación de acciones en los diferentes niveles.

Unafortalezadeestainvestigacióneslaparticipación activa de la Secretaría Distrital de Salud, desde la formulación de la propuesta, hasta la conducción del estudio y el análisis de los resultados, lo cual permite que sean utilizados directamente por los que toman las decisiones para la planeación de los programas y la formulación de las políticas. Esto constituye uno de los aspectos actuales más discutidos en investigación, por la necesidad y las restricciones para crear puentes adecuados entre los científicos y los que formulan las políticas, dadas las diferencias en la oportunidad, el conocimiento entre ellos y la posibilidad de que quienes toman las decisiones se vinculen a los procesos de investigación desde las fases más tempranas, como ocurrió en este caso $(29,30)$.

En particular, el programa en Bogotá podrá orientar acciones hacia el incremento de la captación de pacientes sintomáticos respiratorios en las IPS, mediante la sensibilización del personal médico y el fortalecimiento de la supervisión en las Instituciones prestadoras de servicios de salud.

\section{Conflictos de intereses}

Los autores del presente artículo declaramos que no tenemos conflictos de intereses de orden académico, institucional u operacional en esta investigación.

\section{Financiación}

Estudio financiado por Colciencias, contrato 622104-6548, realizado por la Fundación Santa Fe de Bogotá y la Secretaría de Salud de Bogotá.

\section{Referencias}

1. World Health Organization. WHO Report 2009. Global Tuberculosis Control: surveillance, planning, financing. Geneva: WHO; 2009.

2. Organización Panamericana de la Salud. En 2009, 11.671 casos de tuberculosis en Colombia. Fecha de consulta: mayo 17 de 2010. Disponible en:http://new.paho.org/col/ index.php?option=com_content\&task=view\&id=646\&ltemid $=1 \&$ limit $=1$ \&limitstart $=0$.

3. Secretaría Distrital de Salud de Bogotá. Boletín de prensa. Día mundial de la Tuberculosis. Marzo 24 de 2009. Fecha de consulta: octubre de 2009. Disponible en:http:// www.saludcapital.gov.co/Lists/Anuncion\%20principales/ DispForm.aspx?ID=179.

4. Broekmans J, Paluzzi JE. Meeting the Millennium Development Goals: reversing the global incidence of tuberculosis. Interim report of the TB Working Group within task force five: Infectious diseases and access to essential medicines. New York: United Nations Millennium Project; 2004.

5. World Health Organization. WHO Report 2007. Global Tuberculosis Control: surveillance, planning, financing. Geneva: WHO; 2007.

6. Arciniega A, Barrera MT, Guerrero E. Prevalencia de tuberculosis entre los sintomáticos respiratorios que consultan por primera vez en los establecimientos de salud del departamento de Risaralda, Colombia. Bol Of Sanit Panam. 1979;87:512-24.

7. Acero R, Ibáñez M. Prevalencia de sintomáticos respiratorios y de pacientes con tuberculosis pulmonar en Santafé de Bogotá. Fundación Santa Fe de Bogotá, Secretaría Distrital de Salud. Informe final. Bogotá: Fundación Santa Fe de Bogotá; 1999.

8. García I, De la Hoz F, Reyes Y, Montoya P, Guerrero MI, León Cl. Prevalencia de sintomáticos respiratorios, de infección y enfermedad tuberculosa y factores asociados: estudio basado en población, Mitú, Vaupés, 2001. Biomédica. 2004;24:124-31.

9. Henao SC, Sierra CR, Sánchez EA, Rodríguez AS. Búsqueda de tuberculosis en pacientes sintomáticos respiratorios en cuatro hospitales de Bogotá, D.C. Rev Salud Pública. 2007;9:408-19.

10. Statistics Canada Office. Canada's National Statistical Survey - sampling error. Canada: Canada's National Statistical Agency; 1985.

11. World Health Organization. Treatment of tuberculosis: guidelines for National Programmes. $2^{\text {nd }}$ Edition. TB/97.220. Geneva: wHO; 1997.

12. World Health Organization, Centers for Disease Control and Prevention. Epiinfo: a word processing database and static's program for public health. Versión 6.04. Atlanta: Centers for Disease Control and Prevention; 2000.

13. Stata Inc. Stata 8.0 para Windows. College Station: StataCorp LP; 2004. 
14. Organización Panamericana de la Salud. Control de la tuberculosis. Situación actual. Documentos básicos. Washington, D.C: OPS; 1988.

15. Dye C. Global epidemiology of tuberculosis. Essay focus. Lancet. 2006;367:938-40.

16. Hudelson P. Gender differentials in tuberculosis: The role of socioeconomic and cultural factors. Tuber Lung Dis. 1990;77:391-400.

17. Borgdorff MW, Nagelkerke NJ, Dye C., Nunn P. Gender and tuberculosis: a comparison of prevalence surveys with notification data to explore sex differences in case detection. Int J Tuberc Lung Dis. 2000;4:123-32.

18. World Health Organization. WHO Report 2009 Global Tuberculosis Control: surveillance, planning, financing. Geneva: WHO; 2009.

19. Martínez F, Robayo G, Valencia O. Desarrollo del principio de universalidad en el Sistema General de Seguridad Social en Salud. A ocho años de la reforma en Colombia. Bogotá: Fedesarrrollo; 2002. p. 45-7.

20. Segura AM, Rey JJ, Arbeláez MP. Tendencias de la mortalidad y los egresos hospitalarios por tuberculosis, antes y durante la implementación de la reforma del secto salud, Colombia, 1985-1999. Biomédica. 2004;24:115-23.

21. Arbelaéz MP, Gaviria MB, Franco A, Restrepo R, Incapié D, Blas E. Tuberculosis control and managed competition in Colombia. Int J Health Plann Manag. 2004;19:S25-43.
22. Londoño B, Jaramillo I, Uribe JP. Descentralización y reforma en los servicios de salud: el caso colombiano. Washington D.C.: World Bank; 2001.

23. Machado JE, Martínez JW. Asociación tuberculosis y $\mathrm{VIH}$ en pacientes de Pereira, Colombia. Colomb Med. 2005;36:239-43.

24. Murcia M, Gómez J, Alvarado F, Bustillo J, Mendivelson E, Gómez B. Frequency of tuberculous and non-tuberculous mycobacteria in HIV infected patients from Bogotá, Colombia. BMC Infect Dis. 2001;1:21-7.

25. García I, Merchán A, Chaparro P, López L. Panorama de la coinfección tuberculosis/VIH en Bogotá, 2001. Biomédica. 2004;24:132-7.

26. Castiblanco CA, Ribón W. Coinfección de tuberculosis en pacientes con VIH/SIDA: un análisis según las fuentes de información en Colombia. Infectio. 2006;10:232-42.

27. Victorino C. ¿Cómo mejorar el diagnóstico de la tuberculosis? Rev Chil Enf Respir. 2004;20:181.

28. Chaparro PE, García I, Guerrero MI, León Cl. Situación de la tuberculosis en Colombia, 2002. Biomédica. 2002;4:102-14.

29. Carrasquilla G. Debate on the paper by Celia Almeida \& Ernesto Báscolo. Cad Sáude Pública. 2006;22:24-5.

30. Granada A, Estrada V, Pinto D, Borrero E, Carrasquilla G. Disponibilidad de la información ¿Un cuello de botella para investigación y evaluación en políticas de salud? Rev Salud Pública. 2005;7:201-14. 